\title{
Counterrotation and Barred Galaxies
}

\author{
G. Galletta
}

Dipartimento di Astronomia, Universitá di Padova, vicolo Osservatorio, 5 I 35122 Padova, Italy

\begin{abstract}
In this review we discuss the phenomenon of counterrotation, observed in $\sim 40$ apparently normal galaxies where gas or stars are rotating in a direction opposite to the main stellar body. It appears in all morphological types, from ellipticals to spirals or irregulars.

The problem of the origin and the evolution of counterrotation is discussed, in the wider frame of the galaxies accreting matter from their environment (e.g., minor-axis dust-lane ellipticals or polar-ring S0s). Various sources of matter appear to be candidates for the production of the counterrotation.

Due to the tendency of the bar potential to populate particular families of orbits, some cases of counterrotation found in barred galaxies are interpreted as intrinsic to barred galaxies.
\end{abstract}

\section{Introduction}

We call counterrotation a physical phenomenon for which two parts of a galaxy rotate with opposite spins. This may happen in a portion of the galaxy or in the whole observed range of radii. Assuming as a "reference direction" that described by the rotation of the more massive component of the galaxy (which generally is the stellar body), we can distinguish between gas-or stellar-counterrotation when either the gas (ionized, atomic or molecular) or part of the stars, respectively, is rotating in the opposite direction. The word "counterrotation" was introduced by Franx \& Illingworth (1988) to describe those stellar nuclei of elliptical galaxies rotating in a retrograde way with respect to the outer, more massive stellar body. Today, this term is widely used to define the presence of opposite spins in the same galaxy.

From the observational point of view, the counterrotation produces observable velocities of the two components of the galaxy (or of a portion of it) opposite with respect to the systemic velocity. These opposite motions appear in the same position on the sky and generate velocity curves with typical shapes, described in Figure 1. As we shall show in Sections 5 and 6, the opposite deduction is not always true, i.e. some galaxies exhibit in some spectra opposite motions of gas and stars that do not correspond to opposite spins. 

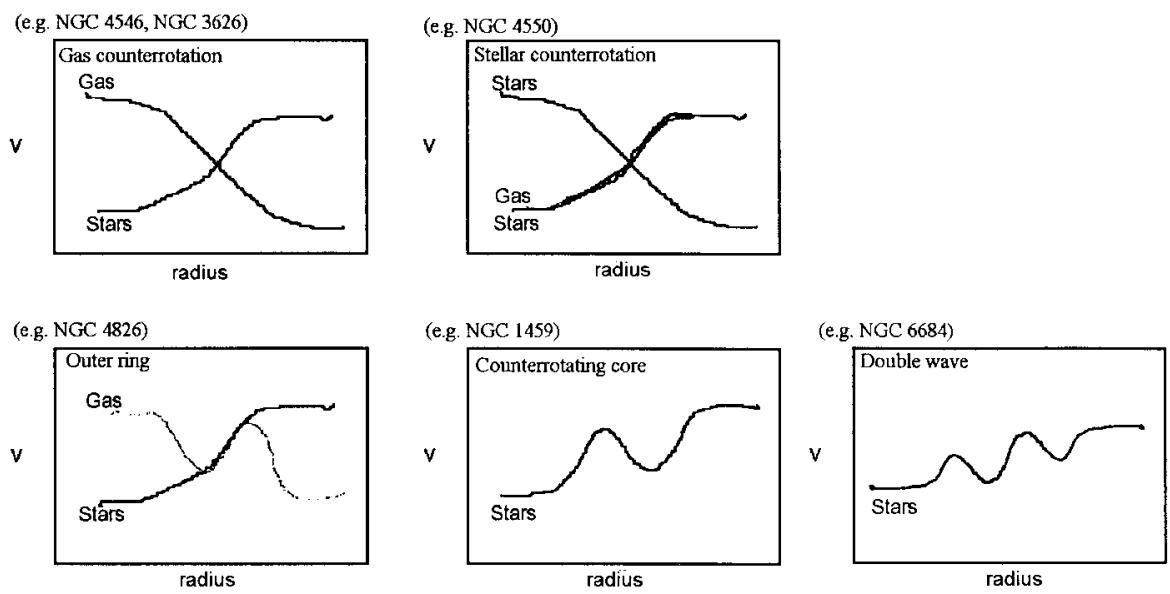

Figure 1. Different kinds of counterrotation, described by means of the observed rotation curves. The extended counterrotations are shown in the top row, while the partial ones are in the bottom row. The galaxy nucleus is set at the center of each graph.

\section{The History and an Inventory of Counterrotations}

The first cases of counterrotation have been discovered for the gas of two elliptical galaxies: NGC 5898 (Bettoni 1984) and NGC 7097 (Caldwell et al. 1986). The feature was so unusual that in both cases the authors spent just a few lines to comment on this peculiarity, suggesting that it was a probable result of the acquisition of gas from the environment.

The gas counterrotation received not much attention until it arose in all its evidence in the first disk galaxy: the barred S0 NGC 4546 (Galletta 1987). Contrary to the above cited elliptical galaxies, here the velocity difference between gas and stars reaches $350 \mathrm{~km} \mathrm{~s}^{-1}$ and was visible when the CCD image of the spectrum was first displayed on the screen! Since 1988, a particular interest in gas counterrotation has been devoted by many astronomers, resulting in an increasing number of papers per year presenting new cases (see Table 1). Seven more objects have been called to our attention during this meeting. After ellipticals and S0s, also a spiral galaxy has been found, NGC 3626, where the whole gas distribution, composed of ionized (Ciri et al. 1995) and atomic hydrogen (van Driel \& van Woerden 1991), is rotating in the opposite direction to the stars. Optical data extend to $3 \mathrm{kpc}$ from center, while $\mathrm{HI}$ data reach $20 \mathrm{kpc}$ from the galaxy nucleus.

The first cases of stellar counterrotation were discovered in 1988 in the nuclei of some elliptical galaxies (Bender 1988, Franx \& Illingworth 1988, Jedrzejewski \& Schechter 1988). Stellar counterrotation in SB0s was found one year later (Bettoni 1989) and finally in the last three years appeared the first cases of 
spiral and S0 galaxies where a percentage (Merrifield \& Kuijken 1994), or even one half of the stars (Rubin et al. 1992, Rix et al. 1992), are counterrotating with respect to the remaining part of the stellar body.

Now we know that all the possible cases of counterrotation exist, considering the type of matter and the morphological type of galaxies. The rule is very simple: "everything you can imagine, it exists". One can find gas counterrotating with respect to stars, stars versus stars, nuclear-stellar body in counterrotation, wavy pattern in stellar rotation curves, central gas co-rotating but counterrotating outside. The phenomenon has been found in every type of galaxy: ellipticals, S0s, spirals and even in irregular galaxies. Concerning the galaxy's environment, it can appear in isolated galaxies as well as in interacting objects, in loose or compact groups, etc. The number of objects with counterrotation reaches today, to our knowledge, 46 cases, presented in Table 1 together with the references discussing the presence of the phenomenon.

\section{Origin of Counterrotation}

After the study of many cases of gas and stellar counterrotations, we are inclined to think that such galaxies are connected to form a wider category of stellar systems, called here generally as gas-accreting galaxies. They are: the minoraxis dust-lane ellipticals, introduced by Bertola \& Galletta (1978) and surveyed by Hawarden et al. (1981), who found dozens of cases; the polar-ring SOs, recognized as a class by Schweizer et al. (1983) and surveyed by Whitmore et al. (1990), who found more than 50 good cases. In these categories of ellipticals and S0s a disk or a ring of gas and stars cross the stellar body perpendicularly to the galaxy apparent major axis. These gas rings or disks are rotating on a plane that is perpendicular to the rotation of the stellar component, a kinematical feature as much peculiar as the counterrotation. More recently, two polar-ring S0s have been demonstrated to be spirals: NGC 660 (van Driel et al. 1995) and NGC 2748 (Bettoni et al. 1996), extending to this morphological type also the detection of polar rings.

Due to the difficulty of believing that the opposite or perpendicular rotation of this matter has been generated by the galaxy itself, it is presumable that this gas (or these stars) derives from the accretion of matter coming from the external regions of the galaxies (Galletta 1987, Whitmore et al. 1990). The gas may enter the system attracted by the gravitational force of the galaxy (the "acceptor" system) and is tidally disrupted and stirred to form a ring (Quinn 1991, Rix \& Katz 1991) and eventually a disk, if dissipative or precessional forces make it to collapse toward the center of the acceptor (Tohline et al. 1982, Steiman-Cameron \& Durisen 1982, Lake \& Norman 1983). The initial spin of the accreted material, if coming from the outside, is independent of the galaxy's spin and can explain the opposite or perpendicular motions observed. In addition, the fact that this process is mainly dependent on the gravitational force justifies the discovery of such features in all the galaxy morphological types.

If the galaxies with counterrotation and the galaxies with polar rings or disks represent the result of the same phenomenon, the number of this more general category of objects increases, and consequently increases the frequency of the gas accretion event among normal galaxies. Whitmore et al. (1990) estimate that 
Table 1. Inventory of the known cases of counterrotation, in order of morphological type and discovery. Various types of counterrotation are known: Gas vs. stars: gas counterrotating with respect to the main stellar body of the galaxy ; Stars vs. stars: stellar counterrotation, in the whole galaxy body ("extended") or confined to the core or intermediate regions; Gas vs. gas: gas in the inner regions rotates in opposite direction with respect to that of the outer regions. The descriptions are illustrated in Figure 1 . Note that the same galaxy may present more than one type of counterrotation. Some cases, labeled with an asterisk, are projected counterrotations but do not correspond to true opposite spins.

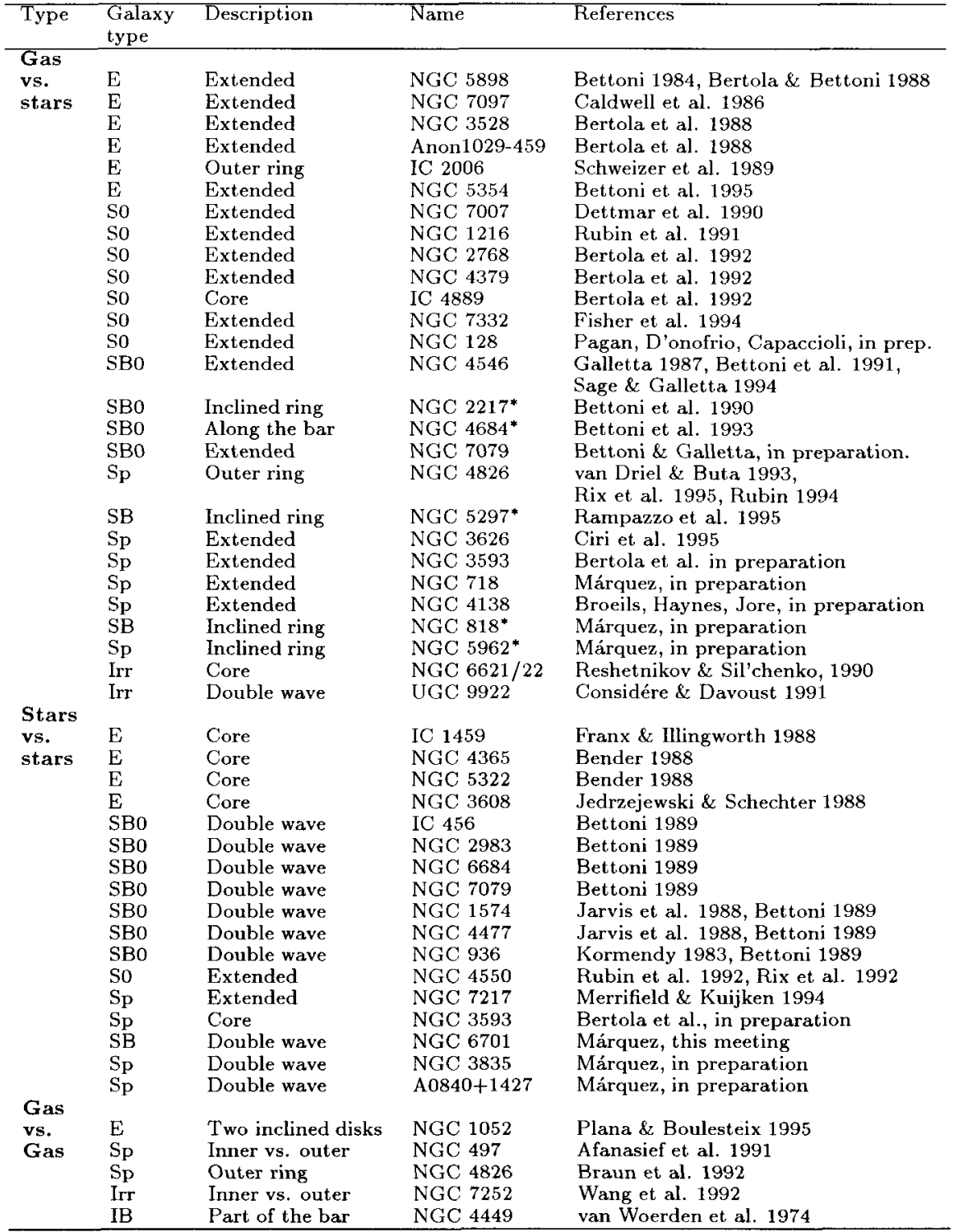


$15 \%$ of S0s may have a polar ring not detectable, due to an unfavorable orientation with respect to the line-of-sight or because the ring is already evolved (or destroyed). Similarly, many cases of counterrotation may be still undiscovered, although a survey of $\sim 15$ edge-on S0s made by Merrifield and Kuijken (private communication) has found no more cases of stellar counterrotation.

If, on one side, the acceptor galaxy may belong to every type of galaxy, we know very little about the source of the accreted gas, that we call here generally the "donor". In the literature, very few theories exist about the generation of counterrotation; the formation of polar rings, widely discussed, is attributed to the capture and disruption of a dwarf companion, maybe an irregular galaxy rich in gas (Toomre 1977, Whitmore et al. 1987), or of dark, gaseous clouds eventually stripped from a close spiral (Whitmore et al. 1987). We note also that the donor's mass must be small with respect to the acceptor, because in most cases the stellar structure of the acceptor is quite regular (disk, bulge and eventually a bar) and the stellar velocity dispersion is similar to that observed in normal galaxies of the same type. A massive donor source should heat the disk and eventually destroy the inner structure, reaching a full merging if donor and acceptor are of equal size. Gas counterrotation is in fact observed in a merging, destroyed system such as NGC 7252, the "Atoms for Peace" galaxy (Wang et al. 1992), and has been simulated by models (Hernquist \& Barnes 1991) obtaining a final structure resembling more an elliptical galaxy than the observed S0s or spirals.

But the recent observations of molecular and atomic gas in minor-axis dustlane ellipticals (see Sage \& Galletta 1993) and in polar ring galaxies (Richter et al. 1994; Galletta et al. 1995) showed the presence of $\mathrm{H}_{2}$ and/or HI masses ranging from $10^{8}$ to $10^{10} \mathrm{M}_{\odot}$ also in galaxies with no close, massive companions. These values are too high for the gas content of a dwarf galaxy, and it is unclear how a bigger donor galaxy may generate counterrotation or a polar structure without heating and destroying the acceptor.

Massive polar rings and massive counterrotations may be explained by the accretion of diffuse matter, due to a secondary infall. The anisotropy of the cosmic mass distribution, on scale lengths typical of galaxy clusters, can generate an angular momentum independent from that of the central galaxy (Voglis et al. 1991). Massive polar rings may be then kept in equilibrium by their own gravitational force (Sparke 1986). The secondary infall cannot yet be due to primordial gas because the observed gas is composed of dust and molecular clouds, a kind of matter chemically evolved from stars. A possible source remains the fall back of the gas processed by the stars and evaporated during the earlier galaxy evolution. When gas or stars are accreted slowly, the new structure should realign itself, while forming, to a common plane with the acceptor's potential. This has been tested, however, for an axisymmetric spheroid only (Binney \& May 1988).

Resuming the above discussion, small quantities of gas accreted in polar planes or in counterrotation may derive from the capture and disruption of a satellite. On the contrary, massive quantities of counterrotating gas (and stars) may derive from a slow gas accretion on the same plane of the galaxy. New studies are required to clarify the gas accretion phenomenon. 


\section{Evolution of Counterrotation}

The evolution of the accreted gas is also unclear. The collapse toward the center, or the shock waves generated by its deformation in a ring (Christodoulou \& Tohline 1991), may induce stellar formation. In some minor-axis dust-lane ellipticals (NGC 1316, NGC 4374 and NGC 5128-Cen A), as well as in the polarring S0s NGC 4650, incipient stellar formation and/or supernova explosions of various types (Barbon et al. 1989) have been observed. No SN explosions have been detected in the galaxies of Table 1, except for NGC 818 .

The stability of the gas in counterrotation is also a problem. Such gas cannot co-exist in the same region of space with gas coeval and co-rotating with stars, and so in the few known cases the two types of gas fill separated regions of the acceptor galaxy (e.g. the outer counterrotating ring in NGC 4826). The collisions with the pre-existing gas should destroy the counterrotation. If there is no pre-existing gas inside the acceptor, but the accreted gas is condensed into clouds, their motions inside a stellar disk happen at a reciprocal velocity of 300 $400 \mathrm{~km} \mathrm{~s}^{-1}$, as observed. The gas should be braked by the dynamical friction (Tremaine 1981) and collapse toward the center. The above mechanisms, as well as the "two stream instability", predict that the counterrotation should be a quite unstable phenomenon. If gas is unstable, a conversion in counterrotating stars or a confinement in an external ring may be expected. In such a case, the stellar counterrotation observed could be simply the residual of previous cases of gas counterrotation. It is difficult to imagine, as an alternative, a pure stellar infall from the environment, or a stellar formation in outer, low density regions during a secondary infall of primordial gas. When stars are formed, differently from plasma instabilities, stellar dynamics can produce under particular conditions stable co-spatial stellar disks in counterrotation (Sellwood \& Merritt 1994).

Looking at the Table 1, galaxies with extended stellar counterrotation (NGC 4550, NGC 7217) may be the final stage of galaxies with gas counterrotation, such as NGC 4546 or NGC 3626 . Also ellipticals with counterrotating cores may result from the stars formed from accreted gas condensed in the system barycenter. Intermediate cases can be found in galaxies where the inner region is already collapsed to the center (NGC 4826, see Rix et al. 1995) or where the central region is dominated by stars in counterrotation; these may be the product of recent stellar formation (NGC 3593). Obviously this is just an attempt to explain these categories of galaxies in the frame of a single physical process, but many aspects are supported by observations and theories. The presence of so many cases of gas counterrotation still remain, in some way, a challenge to the theories.

\section{False Gas Counterrotation in Barred Galaxies}

A number of cases of counterrotation in Table 1 are observed in barred galaxies. The presence of the bar allows a number of kinematical features very difficult to observe in axisymmetric galaxies. The gas may stream on inclined orbits or in elongated ones. These orbits produce, at some positions on the galaxy image, gas and stellar motions appearing opposite. A typical example is the SB0 NGC 
2217 , an almost face-on galaxy (inclination of $\sim 21^{\circ}$ ) where the gas along the bar minor-axis and at intermediate angles shows motions opposite to that of the stars. A detailed analysis shows, however, that this feature agrees with the presence of a warped gas disk rotating in the same direction of the stars. This disk is almost perpendicular to the bar major axis in the inner regions, and warps toward the main galaxy plane in the outer regions. The particular orientation of the observer, with the galaxy seen almost face-on and the gas disk seen almost edge-on, produces the apparent counterrotation observed (see the Figure 6 in Bettoni et al. 1990). Similar cases are suspected in the barred spirals NGC 818 and in the ringed spiral NGC 5962 where the minor-axis spectra present gas counterrotation, and in the SB galaxy NGC 5297. Models of gas orbits in a barred potential were produced by Friedli \& Benz (1993), taking into account the vertical instabilities. They show that gas becoming vertically unstable can be trapped in inclined structures, appearing as counterrotating rings.

In other barred galaxies, the gas moves in elongated orbits with major axes aligned with the bar. In the case of NGC 4684, two streams of ionized gas have been observed that appear to have velocities opposite to that of the stars. This galaxy is an edge-on S0 with a quite small bar, difficult to observe. In $\mathrm{H} \alpha$ light, the nucleus appears surrounded by an oval, luminous disk, $680 \mathrm{pc}$ wide, from which depart two filaments of ionized gas, extending $\sim 3 \mathrm{kpc}$ at the same P.A. of the bar. A mapping of gas and stellar velocities reveals that the apparent opposite motions are not due to opposite rotation, but to some kind of radial motion (inflow or outflow). Models suggest that gas orbiting inside a bar can collapse and loose angular momentum on each passage through the bar and associated shock wave (Friedli et al. 1991). The filaments of NGC 4684 may represent this phenomenon. It allows the formation of a central concentration of matter that tends to destroy the bar (Hasan \& Norman 1990). The inner oval detected in $\mathrm{H} \alpha$ looks like this foreseen concentration of matter.

In both cases, we deduce that the rotation curves presenting opposite motions of gas and stars do not correspond to true counterrotations. In doubtful cases, a mapping of the gas and star velocity field can solve the ambiguity.

\section{Intrinsic Counterrotation in Barred Galaxies}

Looking at Table 1, the cases of partial stellar counterrotation in S0s are present almost exclusively in barred systems. This is due also to the fact that most of the cases were found by Bettoni (1989) and Jarvis et al. (1988) during two surveys of barred galaxies. In the SB0s listed in Table 1 a part of the bar shows stellar motions with a shape indicated in Figure 1 as "double wave" (Bettoni 1989). This wavy pattern of the bar rotation curve is generally superposed on the main galaxy rotation. When the bar is near the apparent galaxy minor axis (perpendicular to the line-of-nodes) the observed velocities actually reverse direction with respect to the nucleus. When the bar is seen closer to the major axis of the galaxy image (the line of the nodes), the galaxy rotation curve is deformed by this "wave", with oscillation of $20-30 \mathrm{~km} \mathrm{~s}^{-1}$, symmetric on the two sides of the bar.

Here again, we can suspect that the complex patterns assumed by the stellar orbits in elongated potentials play the major role in determining this effect. In 
fact, this feature appears in some N-body (Sparke \& Sellwood 1987) and in self-consistent models of bars (Pfenniger 1984). An explanation of this effect as intrinsic to barred galaxies has been proposed by Wozniak (these proceedings). According to this discussion, the stellar counterrotation found in barred S0s should not have an external origin but is a phenomenon caused by the bar.

\section{Conclusions}

The phenomenon of counterrotation is observed today in a not negligible number of systems; it has been discussed in the frame of the wider class of gas-accreting galaxies, as minor-axis dust-lane ellipticals or polar-ring SOs. The origin of the galaxies with counterrotation may be due to different physical processes, producing the same final morphology: 1) satellite accretion and disruption; 2) tidal stripping of gas from a nearby companion; 3) secondary infall of diffuse, non-primordial, gas. Stellar counterrotation, both extended in the whole galaxy or confined to the core, may be the result of past gas accretion, whose instability triggered star formation.

On the contrary, when confined in a portion of a barred galaxy ("double wave" effect), the stellar counterrotation seems to be an intrinsic phenomenon, due to the particular kind of orbits allowed in an elongated potential. The bar allows us also to observe cases of opposite rotation curves confined to particular positions inside the galaxy (bar minor axis or along the bar axis). These false counterrotations may be discovered by a mapping of the galaxy velocity fields (stars and gas) and are also due to the presence of the bar.

\section{References}

Afanasief, A. V., Burenkov, A. N., Zasov, A. V., \& Sil'chenko, O. K. 1991, Astronomicheskii Zhurnal, 68, 1134

Barbon, R., Cappellaro, E., \& Turatto, M. 1989, A\&AS, 81, 421

Bender, R. 1988, A\&A, 202, L5

Bertola, F. \& Bettoni, D. 1988, ApJ, 329, 102

Bertola, F. \& Galletta, G. 1978, ApJ, 226, L115

Bertola, F., Buson, L. M., \& Zeilinger, W. W. 1988, Nature, 335, 705

Bertola, F., Buson, L. M., \& Zeilinger, W. W. 1992, ApJ, 401, L79

Bettoni, D. 1984, The Messenger, 37, 17

Bettoni, D. 1989, AJ, 97, 79

Bettoni, D., Buson, L. M., Maira, L., \& Bertola, F. 1995, Groups of Galaxies, O.-G. Richter \& K. Borne, San Francisco: ASP, in press

Bettoni, D., Ciri, R., Galletta, G., \& Nuzzo, R. 1996, in preparation

Bettoni, D., Fasano, G, \& Galletta, G. 1990, AJ, 99, 1789

Bettoni, D., Galletta, G., \& Oosterloo,T. 1991, MNRAS, 248, 544

Bettoni, D., Galletta, G., \& Sage, L. J. 1993, A\&A, 280, 121

Binney, J. \& May, A. 1988, MNRAS, 218, 743

Braun, R., Walterbos, R. A. M., \& Kennicutt, R. C. 1992, Nature, 360, 442 
Caldwell, N., Kirshner,R. P., \& Richstone, D. O. 1986, ApJ, 305, 136

Christodoulou, D. M. \& Tohline, J. E. 1991 in Warped Discs and Inclined Rings around Galaxies, S. Casertano, P. Sacket, \& F. Briggs, Cambridge: Cambridge Univ. Press, 122

Ciri, R., Bettoni, D., \& Galletta, G. 1995, Nature, 375, 661

Considére, S. \& Davoust, E. 1991, A\&A, 252, 56

Dettmar, R. J., Jullien-Dettmar, M., \& Barteldrees, A. 1990, in The Interstellar Medium in External Galaxies, D. J. Hollenbach \& H. A. Thronson, NASA CP-3084, 246

Fisher, D., Illingworth, G., \& Franx, M. 1994, AJ, 107, 160

Franx, M. \& Illingworth, G. 1988, ApJ, 327, L.55

Friedli, D. \& Benz, W. 1993, A\&A, 268, 65

Friedli, D., Benz, W., \& Martinet, L. 1991, in Dynamics of Disc Galaxies, B. Sundelius, Göteborg: Göteborgs University press, 181

Galletta, G. 1987, ApJ, 318, 531

Galletta, G., Sage, L. J., Sparke, L. S., \& Sackett, P. D. 1995, in preparation.

Hawarden, T. G., Elson, R. A. W., Longmore, A. J., Tritton, S. B., \& Corwin, H. G. 1981, MNRAS, 196, 747

Hasan, H. \& Norman, C. 1990, ApJ, 344, 204

Hernquist, L. \& Barnes, J. E. 1991, Nature, 354, 210

Jarvis, B. J., Dubath, L., Martinet, R., \& Bacon, R. 1988, A\&AS, 74, 513

Jedrzejewski, R. \& Schechter, P. L. 1988, ApJ, 330, L87

Kormendy, J. 1983, ApJ, 275, 529

Lake, G. \& Norman, C. 1983, ApJ, 270, 51

Merrifield, M. \& Kuijken, K. 1994, ApJ, 432, 575

Pfenniger, D. 1984, A\&A, 134, 373

Plana, H. \& Boulesteix, J. 1995, 3D optical Spectroscopy Methods in Astronomy, G. Comte \& M. Marcelin, San Francisco: ASP, 133

Quinn, T. 1991, in Warped Discs and Inclined Rings around Galaxies, S. Casertano, P. Sackett, \& F. Briggs, Cambridge: Cambridge Univ. Press, 143

Rampazzo, R., Reduzzi, L., Sulentic, J. W., \& Madejsky, R. 1995, A\&AS, 110, 1

Reshetnikov, V. P. \& Sil'chenko, O. K. 1990, Astrophysika, 33, 158

Richter, O.-G., Sackett, P. D., \& Sparke, L. S. 1994, AJ, 107, 99

Rix, H.-W. \& Katz, N. 1991, Warped Discs and Inclined Rings around Galaxies, S. Casertano, P. Sacket, \& F. Briggs, Cambridge: Cambridge Univ. Press, 112

Rix, H.-W., Franx, M., Fisher, D., \& Illingworth, G. 1992, ApJ, 400, L5

Rix, H.-W., Kennicutt, R. C., Braun, R., \& Walterbos, R. A. M. 1995, ApJ, 438,155

Rubin, V. C. 1994, AJ, 107, 173

Rubin, V. C., Hunter, D. A., \& Ford, W. K. 1991, ApJS, 76, 153

Rubin, V. C., Graham, J. A., \& Kenney, J. D. P. 1992, ApJ, 394, L9 
Sage, L. J. \& Galletta, G. 1993, ApJ, 419, 544

Sage, L. J. \& Galletta, G. 1994, AJ, 108, 1633

Schweizer, F., Whitmore, B. C., \& Rubin, V. C. 1983, AJ, 88, 909

Schweizer, F., van Gorkom, J. H., \& Seitzer, P. 1989, ApJ, 338, 770

Sellwood, J. A. \& Merritt, D. 1994, ApJ, 425, 530

Sparke, L. S. 1986, MNRAS, 219, 657

Sparke, L. S. \& Sellwood, J. A. 1987, MNRAS, 225, 653

Steiman-Cameron, T. Y. \& Durisen, R. H. 1982, ApJ, 263, L51

Tohline, J. E., Simonson, G. F., \& Caldwell, N. 1982, ApJ, 252, 92

Toomre, A. 1977, in Evolution of Galaxies and Normal Stellar Populations, B. M. Tinsley \& R. B. Larson, New Haven: Yale Univ. Obs., 401

Tremaine, S. 1981, The Structure and Evolution of Normal Galaxies, S. M. Fall \& D. Lynden-Bell, Cambridge: Cambridge Univ. Press, 67

van Woerden, H., Bosma, A., \& Mebold, U. 1974, La Dynamique des Galaxies Spirales, Weliachew, C.N.R.S. coll. 241, 483

van Driel, W. \& Buta, R. 1993, PASJ, 45, L47

van Driel, W., Combes, F., Casoli, F., Gerin, M., Nakai, M., Miyaji, T., Hamabe, M. et al. 1995, AJ, 109, 942

Voglis, N., Hiotelis, N., \& Höflich, P. 1991, A\&A, 249, 5

Whitmore, B. C., McElroy, D. B., \& Schweizer, F. 1987, ApJ, 314, 439

Whitmore, B. C., Lucas, R. A., McElroy, D. B., Steiman-Cameron, T. Y., Sackett, P. D., \& Olling, R. B. 1990, AJ, 100, 1489

Wang, Z., Schweizer, F., \& Scoville, N. Z. 1992, ApJ, 396, 510 